\title{
Weighted Zeta Functions of Graph Coverings
}

\author{
Iwao SATO \\ Oyama National College of Technology, \\ Oyama, Tochigi 323-0806, JAPAN \\ e-mail: isato@oyama-ct.ac.jp
}

Submitted: Jan 7, 2006; Accepted: Oct 10, 2006; Published: Oct 27, 2006

Mathematical Subject Classification: 05C50, 15A15

\begin{abstract}
We present a decomposition formula for the weighted zeta function of an irregular covering of a graph by its weighted $L$-functions. Moreover, we give a factorization of the weighted zeta function of an (irregular or regular) covering of a graph by equivalence classes of prime, reduced cycles of the base graph. As an application, we discuss the structure of balanced coverings of signed graphs.
\end{abstract}

\section{Introduction}

In our previous paper [11], we defined the weighted zeta function and the weighted $L$ function of a graph, and presented their determinant expressions. Furthermore, we expessed the weighted zeta function of a regular covering of a graph by a product of its weighted $L$-functions. In this paper, we study a decomposition formula for the weighted zeta function of an irregular covering of a graph by its weighted $L$-functions. Moreover, we treat a factorization of the weighted zeta function of an (irregular or regular) covering of a graph by equivalence classes of prime, reduced cycles of the base graph. By using the second result, we discuss the structure of balanced coverings of signed graphs.

Graphs and digraphs treated here are finite and simple. Let $G=(V(G), E(G))$ be a connected graph with vertex set $V(G)$ and edge set $E(G)$, and $D$ the symmetric digraph corresponding to $G$. Set $D(G)=\{(u, v),(v, u) \mid u v \in E(G)\}$. For $e=(u, v) \in D(G)$, let $o(e)=u$ and $t(e)=v$. The inverse arc of $e$ is denoted by $e^{-1}$. A path $P$ of length $n$ in $G$ is a sequence $P=\left(v_{0}, v_{1}, \cdots, v_{n-1}, v_{n}\right)$ of $n+1$ vertices and $n$ arcs such that consecutive vertices share an arc (we do not require that all vertices are distinct). Also, $P$ is called a $\left(v_{0}, v_{n}\right)$-path. If $e_{i}=\left(v_{i-1}, v_{i}\right)$ for $i=1, \cdots, n$, then we can write $P=\left(e_{1}, \cdots, e_{n}\right)$. We say that a path has a backtracking if a subsequence of the form $\cdots, x, y, x, \cdots$ appears. A $(v, w)$-path is called a $v$-cycle (or $v$-closed path) if $v=w$. The inverse cycle of a cycle $C=\left(v, v_{1}, \cdots, v_{n}, v\right)$ is the cycle $C^{-1}=\left(v, v_{n}, \cdots, v_{1}, v\right)$. 
We introduce an equivalence relation between cycles. Two cycles $C_{1}=\left(v_{1}, \cdots, v_{m}\right)$ and $C_{2}=\left(w_{1}, \cdots, w_{m}\right)$ are called equivalent if $w_{j}=v_{j+k}$ for all $j$. Let $[C]$ be the equivalnce class which contains a cycle $C$. Let $B^{r}$ be the cycle obtained by going $r$ times around a cycle $B$. Such a cycle is called a multiple of $B$. A cycle $C$ is called reduced if both $C$ and $C^{2}$ have no backtracking. A cycle $C$ is prime if $C \neq B^{r}$ for any $r \geq 2$ and any other cycle $B$. Note that each equivalence class of prime, reduced cycles of a graph $G$ corresponds to a unique conjugacy class of the fundamental group $\pi_{1}(G, v)$ of $G$ at a vertex $v$ of $G$.

The (Ihara) zeta function of a graph $G$ is defined to be a function of $u \in \mathbf{C}$ with $|u|$ sufficiently small, by

$$
\mathbf{Z}(G, u)=\mathbf{Z}_{G}(u)=\prod_{[C]}\left(1-u^{|C|}\right)^{-1}
$$

where $[C]$ runs over all equivalence classes of prime, reduced cycles of $G$, and $|C|$ is the length of $C$.

Zeta functions of graphs started from zeta functions of regular graphs by Ihara [8]. In [8], he showed that their reciprocals are explicit polynomials. A zeta function of a regular graph $G$ associated to a unitary representation of the fundamental group of $G$ was developed by Sunada $[17,18]$. Hashimoto [7] treated multivariable zeta functions of bipartite graphs. Bass [1] generalized Ihara's result on the zeta function of a regular graph to an irregular graph, and showed that its reciprocal is a polynomial:

$$
\mathbf{Z}(G, u)^{-1}=\left(1-u^{2}\right)^{r-1} \operatorname{det}\left(\mathbf{I}-u \mathbf{A}(G)+u^{2}(\mathbf{D}-\mathbf{I})\right),
$$

where $r$ and $\mathbf{A}(G)$ are the Betti number and the adjacency matrix of $G$, respectively, and $\mathbf{D}=\left(d_{i j}\right)$ is the diagonal matrix with $d_{i i}=\operatorname{deg} v_{i}\left(V(G)=\left\{v_{1}, \cdots, v_{n}\right\}\right)$.

Stark and Terras [14] gave an elementary proof of Bass' Theorem, and discussed three different zeta functions of any graph. Furthermore, various proofs of Bass' Theorem were given by Foata and Zeilberger [3], Kotani and Sunada [9]. Stark and Terras [15], and, independently, Mizuno and Sato [10] showed that the zeta function of a regular covering of $G$ is a product of $L$-functions of $G$. Stark and Terras [16] treated zeta functions of bipartite coverings of graphs. Feng, Kwak and Kim [2] gave a decomposition formula for zeta functions of irregular coverings of graphs.

Let $G$ be a connected graph and $V(G)=\left\{v_{1}, \cdots, v_{n}\right\}$. Then we consider an $n \times n$ matrix $\mathbf{W}=\left(w_{i j}\right)_{1 \leq i, j \leq n}$ with $i j$ entry the complex variable $w_{i j}$ if $\left(v_{i}, v_{j}\right) \in D(G)$, and $w_{i j}=0$ otherwise. Furthermore, assume that $w_{j i}^{-1}=w_{i j}$ if $\left(v_{i}, v_{j}\right) \in D(G)$. The matrix $\mathbf{W}=\mathbf{W}(G)$ is called the weighted matrix of $G$. For each path $P=\left(v_{i_{1}}, \cdots, v_{i_{r}}\right)$ of $G$, the norm $w(P)$ of $P$ is defined as follows: $w(P)=w_{i_{1} i_{2}} w_{i_{2} i_{3}} \cdots w_{i_{r-1} i_{r}}$. Furthermore, let $w\left(v_{i}, v_{j}\right)=w_{i j}, v_{i}, v_{j} \in V(G)$, and let $w(e)=w\left(v_{i}, v_{j}\right), e=\left(v_{i}, v_{j}\right) \in D(G)$.

Let $\Gamma$ a finite group and $\alpha: D(G) \longrightarrow \Gamma$ an ordinary voltage assignment. Furthermore, let $\rho$ be a representation of $\Gamma$ and $d$ its degree. For each path $P=\left(v_{1}, \cdots, v_{r}\right)$ of $G$, let $\alpha(P)=\alpha\left(v_{1}, v_{2}\right) \alpha\left(v_{2}, v_{3}\right) \cdots \alpha\left(v_{r-1}, v_{r}\right)$. This is called the net voltage of $P$. Then the weighted $L$-function of $G$ associated to $\rho$ and $\alpha$ is defined by

$$
\mathbf{Z}(u, \rho, \alpha, w)=\mathbf{Z}_{G}(u, \rho, \alpha, w)=\prod_{[C]} \operatorname{det}\left(\mathbf{I}_{d}-\rho(\alpha(C)) w(C) u^{|C|}\right)^{-1},
$$


where $[C]$ runs over all equivalence classes of prime, reduced cycles of $G$.

If $\rho=\mathbf{1}$ is the identity representation of $\Gamma$, then the weighted $L$-function of $G$ associated to $\mathbf{1}$ and $\alpha$ is the (vertex) weighted zeta function of $G$ :

$$
\mathbf{Z}_{G}(w, u)=\prod_{[C]}\left(1-w(C) u^{|C|}\right)^{-1}
$$

where $[C]$ runs over all equivalence classes of prime, reduced cycles of $G$ (see [11]). If $w\left(v_{i}, v_{j}\right)=1$ for each $\left(v_{i}, v_{j}\right) \in D(G)$, then the weighted zeta function of $G$ is the (Ihara) zeta function of $G$.

Mizuno and Sato [11] presented a determinant expression for the weighted zeta function and the weighted $L$-function of a graph $G$.

Let $\mathbf{W}=\mathbf{W}(G)$ be a weighted matrix of $G$.

The Kronecker product $\mathbf{A} \otimes \mathbf{B}$ of square matrices $\mathbf{A}$ and $\mathbf{B}$ is considered as the matrix A having the element $a_{i j}$ replaced by the matrix $a_{i j} \mathbf{B}$.

Theorem 1 (Mizuno and Sato) Let $G$ be a connected graph with $n$ vertices and $m$ edges, $\Gamma$ a finite group and $\alpha: D(G) \longrightarrow \Gamma$ an ordinary voltage assignment. Furthermore, let $\rho$ be a representation of $\Gamma$, and $d$ the degree of $\rho$. Suppose that $G^{\alpha}$ is connected. Then the reciprocal of the weighted $L$-function of $G$ associated to $\rho$ and $\alpha$ is

$$
\mathbf{Z}_{G}(u, \rho, \alpha, w)^{-1}=\left(1-u^{2}\right)^{(m-n) d} \operatorname{det}\left(\mathbf{I}_{d n}-u\left(\sum_{g \in \Gamma} \rho(g) \bigotimes \mathbf{W}_{g}\right)+u^{2}(d \circ \mathbf{Q})\right),
$$

where the matrix $\mathbf{W}_{g}=\left(w_{u v}^{(g)}\right)_{u, v \in V(G)}$ is given by

$$
w_{u v}^{(g)}= \begin{cases}w(u, v) & \text { if }(u, v) \in D(G) \text { and } \alpha(u, v)=g, \\ 0 & \text { otherwise, }\end{cases}
$$

and $\mathbf{Q}=\mathbf{D}-\mathbf{I}, d \circ \mathbf{Q}=\mathbf{I}_{d} \otimes \mathbf{Q}$.

Specially, in the case of $\rho=\mathbf{1}$, the reciprocal of the weighted zeta function of $G$ is given by

$$
\mathbf{Z}_{G}(w, u)^{-1}=\left(1-u^{2}\right)^{m-n} \operatorname{det}\left(\mathbf{I}-u \mathbf{W}(G)+u^{2} \mathbf{Q}\right) .
$$

Mizuno and Sato [11] showed that the weighted zeta function of a regular covering of $G$ is a product of weighted $L$-functions of $G$. We are interested in factorizing the weighted zeta function of an irregular covering of a graph by its weighted $L$-functions. In this paper, we prove the following theorem.

Main Theorem 1 Let $G$ be a connected graph, $S_{n}$ the symmetric group on $N=\{1,2, \ldots$, $n\}, \alpha: D(G) \longrightarrow S_{n}$ a permutation voltage assignment and $\mathbf{W}=\mathbf{W}(G)$ a weighted matrix of $G$. Then the weighted zeta function of $G^{\alpha}$ is given by

$$
\mathbf{Z}_{G^{\alpha}}(\tilde{w}, u)=\prod_{\rho} \mathbf{Z}_{G}(u, \rho, \alpha, w)^{m_{\rho}}
$$

where $\rho$ runs over all inequivalent irreducible representations of $\Gamma=<\{\alpha(u, v) \mid(u, v) \in$ $D(G)\}>$, and $m_{\rho}$ is the multiplicity of $\rho$ in the permutation representation of $\Gamma$. 
Furthermore, we express the weighted zeta function of an (irregular or regular) covering of a graph in the Euler product in terms of equivalence classes of prime, reduced cycles of the base graph(c.f., [12]).

In the case that $\Gamma=\{1,-1$,$\} is the cyclic group of order 2$, the ordinary voltage graph $(G, w)$ for an ordinary voltage assignment $w: D(G) \longrightarrow \mathbf{Z}_{2}$ is a signed graph. A balanced signed graph is a generalization of a bipartite graph. The factorization of the weighted zeta function of a covering by equivalence classes of prime, reduced cycles of the base graph might be used to study the structure of a balanced covering of a signed graph. Actually, Stark and Terras [16] studied the location of the poles of zeta and $L$-functions of graphs, and obtained the following result on the structure of bipartite coverings of graphs.

Theorem 2 (Stark and Terras) Let $X$ be a finite connected graph with rank $k \geq 1$ and $Y$ a bipartite covering of $X$.

1. When $X$ is bipartite, every intermediate covering $\tilde{X}$ to $Y / X$ is bipartite.

2. When $X$ is not bipartite, there is a unique quadratic covering $X_{2}$ intermediate to $Y / X$ such that any intermediate covering $\tilde{X}$ to $Y / X$ is bipartite if and only if $\tilde{X}$ is intermediate to $Y / X_{2}$.

We present an analogue of Theorem 2 for balanced coverings of signed graphs.

In Section 2, we present a decomposition formula for the weighted zeta function of an irregular covering of a graph $G$ by weighted $L$-functions of $G$. Furthermore, we give another decomposition formula for the weighted zeta function of an irregular or a regular covering of a graph $G$ by using equivalence classes of prime, reduced cycles of $G$. In Section 3 , we discuss the structure of balanced coverings of $G$ by using the second decomposition formula for the weigthed zeta function of a covering of $G$.

For a general theory of the representation of groups and graph coverings, the reader is referred to [13] and [5], respectively.

\section{Decomposition formulas for weighted zeta func- tions of graph coverings}

Let $G$ be a connected graph, and let $N(v)=\{w \in V(G) \mid(v, w) \in D(G)\}$ denote the neighbourhood of a vertex $v$ in $G$. A graph $H$ is called a covering of $G$ with projection $\pi: H \longrightarrow G$ if there is a surjection $\pi: V(H) \longrightarrow V(G)$ such that $\left.\pi\right|_{N\left(v^{\prime}\right)}: N\left(v^{\prime}\right) \longrightarrow N(v)$ is a bijection for all vertices $v \in V(G)$ and $v^{\prime} \in \pi^{-1}(v)$. The projection $\pi: H \longrightarrow G$ is an $n$-fold covering of $G$ if $\pi$ is $n$-to-one. When a finite group $\Pi$ acts on a graph $G$, the quotient graph $G / \Pi$ is a simple graph whose vertices are the $\Pi$-orbits on $V(G)$, with two vertices adjacent in $G / \Pi$ if and only if some two of their representatives are adjacent in $G$. A covering $\pi: H \longrightarrow G$ is said to be regular if there is a subgroup $B$ of the automorphism group Aut $H$ of $H$ acting freely on $H$ such that the quotient graph $H / B$ is isomorphic to $G$. 
Let $G$ be a graph, $\Gamma$ a finite group and $S_{n}$ the symmetric group on the set $N=$ $\{1,2, \cdots, n\}$. Then a mapping $\alpha: D(G) \longrightarrow S_{n}(\alpha: D(G) \longrightarrow \Gamma)$ is called a permutation voltage assignment (an ordinary voltage assignment) if $\alpha(v, u)=\alpha(u, v)^{-1}$ for each $(u, v) \in$ $D(G)$. The pair $(G, \alpha)$ is called a permutation voltage graph (an ordinary voltage graph) for a permutation voltage assignment (an ordinary voltage assignment) $\alpha$. The derived graph $G^{\alpha}$ of the permutation (ordinary) voltage graph $(G, \alpha)$ is defined as follows: $V\left(G^{\alpha}\right)=$ $V(G) \times N\left(V\left(G^{\alpha}\right)=V(G) \times \Gamma\right)$ and $((u, h),(v, k)) \in D\left(G^{\alpha}\right)$ if and only if $(u, v) \in D(G)$ and $k=\alpha(u, v)(h)(k=h \alpha(u, v))$. The natural projection $\pi_{\alpha}: G^{\alpha} \longrightarrow G$ is defined by $\pi_{\alpha}(u, h)=u$. The graph $G^{\alpha}$ is called a derived graph covering of $G$ with voltages in $S_{n}$ (or $\Gamma$ ) or an $n$-covering (or a $\Gamma$-covering) of $G$. Note that the $n$-covering ( $\Gamma$-covering) $G^{\alpha}$ is an $n$-fold (regular) covering of $G$. Futhermore, every $n$-fold (regular) covering of a graph $G$ is an $n$-covering ( $\Gamma$-covering) $G^{\alpha}$ of $G$ for some permutation (ordinary) voltage assignment $\alpha: D(G) \longrightarrow S_{n}(\alpha: D(G) \longrightarrow \Gamma)($ see $[4,5])$.

Let $G$ be a connected graph, $S_{n}$ the symmetric group on $N=\{1,2, \cdots, n\}$ ( $\Gamma$ a finite group) and $\alpha: D(G) \longrightarrow S_{n}$ a permutation voltage assignment $(\alpha: D(G) \longrightarrow \Gamma$ an ordinary voltage assignment). In the $\Gamma$-covering $G^{\alpha}$, set $v_{g}=(v, g)$ and $e_{g}=(e, g)$, where $v \in V(G), e \in D(G), 1 \leq g \leq n(g \in \Gamma)$. For $e=(u, v) \in D(G)$, the arc $e_{g}$ emanates from $u_{g}$ and terminates at $v_{\alpha(e)(g)}\left(v_{g \alpha(e)}\right)$. Note that $e_{g}^{-1}=\left(e^{-1}\right)_{\alpha(e)(g)}\left(e_{g}^{-1}=\left(e^{-1}\right)_{g \alpha(e)}\right)$.

Let $\mathbf{W}=\mathbf{W}(G)$ be a weighted matrix of $G$. Then we define the weighted matrix $\tilde{\mathbf{W}}=\mathbf{W}\left(G^{\alpha}\right)=\left(\tilde{w}\left(x_{g}, y_{h}\right)\right)$ of $G^{\alpha}$ derived from $\mathbf{W}$ as follows: $\tilde{w}\left(x_{g}, y_{h}\right)=w(x, y)$ if $(x, y) \in D(G), h=\alpha(x, y)(g)(h=g \alpha(x, y))$ and $\tilde{w}\left(x_{g}, y_{h}\right)=0$ otherwise. Furthermore, let $\tilde{w}_{G^{\alpha}}=\tilde{w}$.

The permutation matrix $\mathbf{P}_{\gamma}=\left(p_{i j}^{(\gamma)}\right)_{1 \leq i, j \leq n}$ of $\gamma \in S_{n}$ over $N$ is defined as follows:

$$
p_{i j}^{(\gamma)}= \begin{cases}1 & \text { if } \gamma(i)=j \\ 0 & \text { otherwise }\end{cases}
$$

Let $\mathbf{M}_{1} \oplus \cdots \oplus \mathbf{M}_{s}$ be the block diagonal sum of square matrices $\mathbf{M}_{1}, \cdots, \mathbf{M}_{s}$. If $\mathbf{M}_{1}=$ $\mathbf{M}_{2}=\cdots=\mathbf{M}_{s}=\mathbf{M}$, then we write $s \circ \mathbf{M}=\mathbf{M}_{1} \oplus \cdots \oplus \mathbf{M}_{s}$.

We present a decomposition formula for the weighted zeta function of an irregular covering of a graph by a product of its weighted $L$-functions.

Theorem 3 Let $G$ be a connected graph with $\nu$ vertices and $\epsilon$ edges, $S_{n}$ the symmetric group on $N=\{1,2, \cdots, n\}, \alpha: D(G) \longrightarrow S_{n}$ a permutation voltage assignment and $\mathbf{W}=\mathbf{W}(G)$ a weighted matrix of $G$. Let $\Gamma=<\{\alpha(u, v) \mid(u, v) \in D(G)\}>$ be the subgroup of $S_{n}$ generated by $\{\alpha(u, v) \mid(u, v) \in D(G)\}$. Furthermore, let $\rho_{1}=1, \rho_{2}, \cdots, \rho_{k}$ be the irreducible representations of $\Gamma$, and $f_{i}$ the degree of $\rho_{i}$ for each $i$, where $f_{1}=1$. Let $P: \Gamma \longrightarrow \operatorname{GL}(n, \mathbf{C})$ be the permutation representation of $\Gamma$ such that $P(\gamma)=\mathbf{P}_{\gamma}$ for $\gamma \in \Gamma$. Suppose that $G^{\alpha}$ is connected, and $m_{i}$ is the multiplicity of $\rho_{i}$ in $P$ for each $i=1, \cdots, k$, that is, $P$ is equivalent to a representation $1 \oplus m_{2} \circ \rho_{2} \oplus \cdots \oplus m_{k} \circ \rho_{k}$. Then the weighted zeta function of $G^{\alpha}$ is

$$
\mathbf{Z}_{G^{\alpha}}(\tilde{w}, u)=\prod_{i=1}^{k} \mathbf{Z}_{G}\left(u, \rho_{i}, \alpha, w\right)^{m_{i}}
$$


Proof . Let $V(G)=\left\{v_{1}, \cdots, v_{\nu}\right\}$. Arrange arcs of $G^{\alpha}$ in $m$ blocks: $\left(v_{1}, 1\right), \cdots,\left(v_{\nu}, 1\right)$; $\left(v_{1}, 2\right), \cdots,\left(v_{\nu}, 2\right) ; \cdots ;\left(v_{1}, n\right), \cdots,\left(v_{\nu}, n\right)$. We consider the weighted matrix $\mathbf{W}\left(G^{\alpha}\right)$ under this order.

Let $\gamma \in \Gamma$. Suppose that $p_{i j}^{(\gamma)}=1$, i.e., $j=\gamma(i)$. If $(u, v) \in D(G)$ and $\alpha(u, v)=\gamma$, then $j=\alpha(u, v)(i)=\gamma(i)$, i.e., $((u, i),(v, j)) \in D\left(G^{\alpha}\right)$ Thus we have

$$
\mathbf{W}\left(G^{\alpha}\right)=\sum_{\gamma \in \Gamma} \mathbf{P}_{\gamma} \otimes \mathbf{W}_{\gamma}
$$

where the matrix $\mathbf{W}_{\gamma}=\left(w_{u v}^{(\gamma)}\right)_{u, v \in V(G)}$ is given as follows: $w_{u v}^{(\gamma)}=w(u, v)$ if $(u, v) \in D(G)$ and $\alpha(u, v)=\gamma$, and $w_{u v}^{(\gamma)}=0$ otherwise.

Let $P: \Gamma \longrightarrow \mathrm{GL}(n, \mathbf{C})$ be the permutation representation of $\Gamma$ such that $P(\gamma)=\mathbf{P}_{\gamma}$. Then there exists a nonsingular matrix $\mathbf{P}$ such that $\mathbf{P}^{-1} P(\gamma) \mathbf{P}=(1) \oplus m_{2} \circ \rho_{2}(\gamma) \oplus \cdots \oplus$ $m_{k} \circ \rho_{k}(\gamma)$ for each $\gamma \in \Gamma$ (see [13]). Putting $\mathbf{B}=\left(\mathbf{P}^{-1} \otimes \mathbf{I}_{\nu}\right) \mathbf{W}\left(G^{\alpha}\right)\left(\mathbf{P} \otimes \mathbf{I}_{\nu}\right)$, we have

$$
\mathbf{B}=\sum_{\gamma \in \Gamma}\left\{(1) \oplus m_{2} \circ \rho_{2}(\gamma) \oplus \cdots \oplus m_{k} \circ \rho_{k}(\gamma)\right\} \otimes \mathbf{W}_{\gamma} .
$$

Note that $\mathbf{W}(G)=\sum_{\gamma \in \Gamma} \mathbf{W}_{\gamma}$ and $1+m_{2} f_{2}+\cdots+m_{k} f_{k}=n$. Therefore it follows that

$$
\begin{aligned}
& \mathbf{Z}_{G^{\alpha}}(\tilde{w}, u)^{-1}=\left(1-u^{2}\right)^{(\epsilon-\nu) n} \operatorname{det}\left(\mathbf{I}_{\nu n}-u \mathbf{W}\left(G^{\alpha}\right)+\left(\mathbf{I}_{n} \otimes\left(\mathbf{D}-\mathbf{I}_{\nu}\right) u^{2}\right)\right) \\
&=\left(1-u^{2}\right)^{\epsilon-\nu} \operatorname{det}\left(\mathbf{I}_{\nu}-u \mathbf{W}(G)+\left(\mathbf{D}-\mathbf{I}_{\nu}\right) u^{2}\right) \\
& \times \prod_{i=2}^{k}\left\{\left(1-u^{2}\right)^{(\epsilon-\nu) f_{i}} \operatorname{det}\left(\mathbf{I}_{\nu f_{i}}-u \sum_{\gamma \in \Gamma} \rho_{i}(\gamma) \otimes \mathbf{W}_{\gamma}+u^{2}\left(\mathbf{I}_{f_{i}} \boldsymbol{\otimes}\left(\mathbf{D}-\mathbf{I}_{\nu}\right)\right)\right\}^{m_{i}} .\right.
\end{aligned}
$$

By Theorem 1, the result follows. Q.E.D.

If $\alpha(e), e \in D(G)$ is considered as a permutation of $S_{\Gamma}$ by the right multiplication $\alpha(e)(g)=g \alpha(e), g \in \Gamma$, then the $\Gamma$-covering $G^{\alpha}$ of $G$ is considered as a $|\Gamma|$-covering of $G$. The group $\left\{\alpha(e) \in S_{\Gamma} \mid e \in D(G)\right\}$ coincides with $\Gamma$. Furthermore, the permutation representation $P: \Gamma \rightarrow \mathrm{GL}(|\Gamma|, \mathbf{C})$ of $\Gamma$ is the right regular representation of $\Gamma$. If $\rho_{1}=1, \rho_{2}, \cdots, \rho_{k}$ are inequivalent irreducible representations of $\Gamma$, then the multiplicity $m_{i}$ of $\rho_{i}$ for $P$ is equal to its degree $f_{i}$ for each $i=1, \cdots, k$.

Then we obtain a decomposition formula for the weighted zeta function of a regular covering of a graph by Mizuno and Sato [11].

Corollary 1 (Mizuno and Sato) Let $G$ be a connected graph, $\Gamma$ a finite group, $\alpha$ : $D(G) \longrightarrow \Gamma$ an ordinary voltage assignment and $\mathbf{W}=\mathbf{W}(G)$ a weighted matrix of $G$. Suppose that the $\Gamma$-covering $G^{\alpha}$ of $G$ is connected. Then we have

$$
\mathbf{Z}_{G^{\alpha}}(\tilde{w}, u)=\prod_{\rho} \mathbf{Z}_{G}(u, \rho, \alpha, w)^{\operatorname{deg} \rho},
$$

where $\rho$ runs over all inequivalent irreducible representations of $\Gamma$. 
Let $\eta$ be a permutation in the symmetric group $S_{n}$. Any permutation has a unique decomposition as a product of disjoint cyclic permutations. For $j=1, \cdots, n$, let $c_{j}$ denote the number of $j$-cycles (cyclic permutations with length $j$ ) in the decomposition of $\eta$. Then $\left(c_{1}, \cdots, c_{n}\right)$ is called the cycle structure of $\eta$.

Theorem 4 Let $G$ be a connected graph, $N=\{1,2, \cdots, n\}$ and $\alpha: D(G) \longrightarrow S_{n}$ a permutation voltage assignment. Let $\mathbf{W}=\mathbf{W}(G)$ be a weighted matrix of $G$. Then the reciprocal of the weighted zeta function of $G^{\alpha}$ is

$$
\mathbf{Z}_{G^{\alpha}}(\tilde{w}, u)^{-1}=\prod_{[C]} \prod_{j=1}^{n}\left(1-w(C)^{j} u^{|C| j}\right)^{c_{j}},
$$

where $[C]$ runs over all equivalence classes of prime, reduced cycles of $G$, and $\left(c_{1}, \cdots, c_{n}\right)$ is the cycle structure of $\alpha(C)$.

Proof. Let $C$ be any prime, reduced cycle of $G^{\alpha}$ and $\pi(C)=C_{0}^{k}$, where $C_{0}$ is a prime, reduced cycle of $G$ and $\pi: G^{\alpha} \longrightarrow G$ is the natural projection. Let $\left(c_{1}, \cdots, c_{n}\right)$ be the cycle structure of $\alpha\left(C_{0}\right)$. By [5, Theorem 2.4.3], the preimage $\pi^{-1}\left(C_{0}\right)$ of $C_{0}$ in $G^{\alpha}$ is the union of $c_{j}$ disjoint cycles with length $j\left|C_{0}\right|$ for each $j=1, \cdots, n$, and so $k=j$ for some $j$ such that $c_{j} \neq 0$. Therefore, it follows that

$$
\mathbf{Z}_{G^{\alpha}}(\tilde{w}, u)^{-1}=\prod_{\left[C_{0}\right]} \prod_{j=1}^{n}\left(1-w\left(C_{0}\right)^{j} u^{|C| j}\right)^{c_{j}},
$$

where $\left[C_{0}\right]$ runs over all equivalence classes of prime, reduced cycles of $G$. Q.E.D.

Let $w_{i j}=1$ unless $w_{i j}=0$. Then we obtain the following result.

Corollary 2 Let $G$ be a connected graph, $N=\{1,2, \cdots, n\}$ and $\alpha: D(G) \longrightarrow S_{n}$ a permutation voltage assignment. Let $\mathbf{W}=\mathbf{W}(G)$ be a weighted matrix of $G$. Then the reciprocal of the zeta function of $G^{\alpha}$ is

$$
\mathbf{Z}\left(G^{\alpha}, u\right)^{-1}=\prod_{[C]} \prod_{j=1}^{n}\left(1-u^{|C| j}\right)^{c_{j}},
$$

where $[C]$ runs over all equivalence classes of prime, reduced cycles of $G$.

We denote the order of an element $g$ of a finite group $\Gamma$ by $\operatorname{ord}(g)$. A similar result to Theorem 4 for a regular covering of a graph is given as follows:

Theorem 5 Let $G$ be a connected graph, $\Gamma$ a finite group with $n$ elements, and $\alpha$ : $D(G) \longrightarrow \Gamma$ an ordinary voltage assignment. Let $\mathbf{W}=\mathbf{W}(G)$ be a weighted matrix of $G$. Then the reciprocal of the weighted zeta function of $G^{\alpha}$ is

$$
\mathbf{Z}_{G^{\alpha}}(\tilde{w}, u)^{-1}=\prod_{[C]}\left(1-w(C)^{\operatorname{ord}(\alpha(C))} u^{|C| \operatorname{ord}(\alpha(C))}\right)^{n / \operatorname{ord}(\alpha(C))},
$$

where $[C]$ runs over all equivalence classes of prime, reduced cycles of $G$. 
Proof. Let $C$ be any prime, reduced cycle of $G^{\alpha}$ and $\pi(C)=C_{0}^{k}$, where $C_{0}$ is a prime, reduced cycle of $G$ and $\pi: G^{\alpha} \longrightarrow G$ is the natural projection. Let $m=\operatorname{ord}\left(\alpha\left(C_{0}\right)\right)$. By [5, Theorem 2.1.3], the preimage $\pi^{-1}\left(C_{0}\right)$ of $C_{0}$ in $G^{\alpha}$ is the union of $n / m$ disjoint cycles with length $m\left|C_{0}\right|$, and so $k=m$. Therefore, it follows that

$$
\mathbf{Z}_{G^{\alpha}}(\tilde{w}, u)^{-1}=\prod_{\left[C_{0}\right]}\left(1-w\left(C_{0}\right)^{\operatorname{ord}\left(\alpha\left(C_{0}\right)\right)} u^{\left|C_{0}\right| \operatorname{ord}\left(\alpha\left(C_{0}\right)\right)}\right)^{n / \operatorname{ord}\left(\alpha\left(C_{0}\right)\right)}
$$

where $\left[C_{0}\right]$ runs over all equivalence classes of prime, reduced cycles of $G$. Q.E.D.

Let $w_{i j}=1$ unless $w_{i j}=0$. Then we obtain a disconnected version of Theorem 1 in $[12]$.

Corollary 3 (Sato) Let $G$ be a connected graph, $\Gamma$ a finite group with $n$ elements, and $\alpha: D(G) \longrightarrow \Gamma$ an ordinary voltage assignment. Then the reciprocal of the zeta function of $G^{\alpha}$ is

$$
Z\left(G^{\alpha}, u\right)^{-1}=\prod_{[C]}\left(1-u^{|C| \operatorname{ord}(\alpha(C))}\right)^{n / \operatorname{ord}(\alpha(C))},
$$

where $[C]$ runs over all equivalence classes of prime, reduced cycles of $G$.

\section{An application of weighted zeta functions of graphs}

We give another notation of a path of a graph $G$. Let $P=\left(v_{0}, v_{1}, \cdots, v_{n-1}, v_{n}\right)=$ $\left(e_{1}, \cdots, e_{n}\right)$ be a path of length $n$ in $G$, where $e_{i}=\left(v_{i-1}, v_{i}\right)$ for $i=1, \cdots, n$. Then we also write $P=\left(v_{0}, e_{1}, v_{1}, e_{2}, v_{2}, \cdots, v_{n-1}, e_{n}, v_{n}\right)$. Furthermore, $v_{0}, v_{1}, \cdots, v_{n-1}, v_{n}$ are called vertices of $P$. A cycle $C=\left(v_{0}, e_{1}, v_{1}, \cdots, v_{n-1}, e_{n}, v_{n}\right)\left(v_{0}=v_{n}\right)$ is called essential if all vertices of $C$ except $v_{0}, v_{n}$ are distinct. An essential cycle is the same as a cycle in standard books on graph theory. Note that any essential cycle is a prime, reduced cycle, and any prime, reduced cycle is a union of disjoint essential cycles.

Let $G$ be a connected graph and $w: D(G) \longrightarrow \mathbf{Z}_{2}=\{ \pm 1\}$ a function such that $w\left(e^{-1}\right)=w(e)$ for any $e \in D(G)$. Then the pair $(G, w)$ is called a signed graph and $w$ is called a sign of $G$ (see [6]). An arc $e$ is called positive(negative) if $w(e)=1(w(e)=-1)$. For a path $P=\left(e_{1}, \cdots, e_{n}\right)$ of $G$, the sign $w(P)$ of $P$ is defined as follows:

$$
w(P)=w\left(e_{1}\right) \cdots w\left(e_{n}\right)
$$

A signed graph $(G, w)$ is balanced if $w(C)=1$ for any essential cycle $C$ of $G$. Otherwise $(G, w)$ is called unbalanced. Note that a signed graph $(G, w)$ is balanced if $w(C)=1$ for any prime, reduced cycle $C$ of $G$.

Harary [6] gave a characterization for a signed graph to be balanced.

Theorem 6 (Harary) Let $G$ be a connected graph and $w: D(G) \longrightarrow \mathbf{Z}_{2}=\{ \pm 1\}$ a sign. Then the following two conditions are equivalent:

1. A signed graph $(G, w)$ is balanced. 
2. Its vertex set can be divided into two sets (possibly empty), $X$ and $Y$, so that each edge between the sets is negative and each edge within a set is positive.

For a signed graph $(G, w)$, we can consider the weighted zeta function $\mathbf{Z}_{G}(w, u)$ of $G$ associated with $w$. The following result is clear.

Proposition 1 Let $G$ be a connected graph and $w: D(G) \longrightarrow \mathbf{Z}_{2}=\{ \pm 1\}$ a sign. If a signed graph $(G, w)$ is balanced, then the weighted zeta funtion of $G$ associated with $w$ is equal to the (Ihara) zeta function of $G: \mathbf{Z}_{G}(w, u)=\mathbf{Z}(G, u)$.

We do not know whether the converse of Proposition 1 holds. By the definition of balanced signed graphs, it is clear that a signed graph $(G, w)$ is balanced if and only if $\mathbf{W}(G)=\mathbf{S A}(G) \mathbf{S}^{-1}$ for some diagonal matrix $\mathbf{S}=\left(s_{i j}\right)$, where $s_{i i}= \pm 1$ for each $i$.

By Bass' Theorem and Theorem 1, we propose the following conjecture.

Conjecture 1 Let $(G, w)$ be a signed graph. Suppose that $G^{w}$ is connected. If

$$
\operatorname{det}\left(\mathbf{I}-u \mathbf{A}(G)+u^{2} \mathbf{Q}\right)=\operatorname{det}\left(\mathbf{I}-u \mathbf{W}(G)+u^{2} \mathbf{Q}\right),
$$

then $(G, w)$ is balanced.

Since a sign $w: D(G) \longrightarrow \mathbf{Z}_{2}$ is an ordinary voltage assignment, $G^{w}$ is a $\mathbf{Z}_{2}$-covering of $G$. By Corollary 2 of [5, Theorem 2.5.1], $G^{w}$ is connected if and only if the local voltage group $\mathbf{Z}_{2}(x)$ at a fixed vertex $x$ of $G$ is equal to $\mathbf{Z}_{2}$, where $\mathbf{Z}_{2}(x)$ is defined as the set of net voltages $w(C)$ on $x$-cycles $C$ in $G$ (see [5]). Thus, $G^{w}$ is connected if and only if there exists a cycle $C$ such that $w(C)=-1$.

Now, let $(G, w)$ be a signed graph of $G$ and $\pi: H \longrightarrow G$ a finite covering of $G$. Then we define the sign $\tilde{w}_{H}: D(H) \longrightarrow\{ \pm 1\}$ of $H$ derived from $w$ as follows:

$$
\tilde{w}_{H}(\tilde{e})=w(e) \text { if } \tilde{e} \in \pi^{-1}(e), e \in D(G) .
$$

We denote the minimum degree of $G$ by $\delta(G)$.

Theorem 7 Let $G$ be a connected graph with $\delta(G) \geq 2, w: D(G) \longrightarrow \mathbf{Z}_{2}=\{ \pm 1\}$ a sign and $\pi: H \longrightarrow G$ any finite covering of $G$. Then the following two results hold:

1. A signed graph $\left(H, \tilde{w}_{H}\right)$ is balanced if $(G, w)$ is balanced.

2. In the case that $(G, w)$ is unbalanced and $\left(H, \tilde{w}_{H}\right)$ is balanced, there exists a unique quadratic covering $G_{2}$ of $G$ such that $\left(K, \tilde{w}_{K}\right)$ is balanced for any intermediate covering $K$ to $H / G$ if and only if $K$ is intermediate covering to $H / G_{2}$.

Proof. 1: If $G$ is blanced, then the partition $V(G)=V_{1} \cup V_{2}$ lifts a partition of $H$ so $H$ is balanced.

2: At first, we consider the double covering $G^{w}$ of $G$. By Theorem 5, we have

$$
\mathbf{Z}_{G}(w, u)^{-1}=\prod_{[C]}\left(1-w(C)^{\operatorname{ord}(w(C))} u^{|C| \operatorname{ord}(w(C))}\right)^{2 / \operatorname{ord}(w(C))},
$$


where $[C]$ runs over all equivalence classes of prime, reduced cycles of $G$. If $w(C)=-1$, then $\operatorname{ord}(w(C))=2$, and so $w(C)^{\operatorname{ord}(w(C))}=1$. Thus, $\left(G^{w}, \tilde{w}_{G^{w}}\right)$ is balanced. Futhermore, a partition of $V\left(G^{w}\right)$ satisfying the condition of Theorem 6 is given as follows:

$$
V_{1}=\left\{v_{1}=(v, 1) \mid v \in V(G)\right\} \text { and } V_{2}=\left\{v_{-1}=(v,-1) \mid v \in V(G)\right\} .
$$

Now, let $V(H)=\tilde{V}_{1} \cup \tilde{V}_{2}$ be a partition of $V(H)$ satisfying the condition of Theorem 6 . Let $H$ be an $n$-fold covering of $G$. Since any regular $n$-fold covering is an $n$-fold covering, we assume that $H$ is irregular. Then there exists a permutation voltage assignment $\alpha: D(G) \longrightarrow S_{n}$ such that $H=G^{\alpha}$. By Theorem 4 and Corollary 2, we have

$$
\prod_{[C]} \prod_{j=1}^{n}\left(1-w(C)^{j} u^{|C| j}\right)^{c_{j}}=\prod_{[C]} \prod_{j=1}^{n}\left(1-u^{|C| j}\right)^{c_{j}},
$$

where $[C]$ runs over all equivalence classes of prime, reduced cycles of $G$, and $\left(c_{1}, \cdots, c_{n}\right)$ is the cycle structure of $\alpha(C)$.

Let $C$ be any prime, reduced cycle of $G$ such that $W(C)=-1$. Then $j$ is even if $c_{j} \neq 0(1 \leq j \leq n)$. By [5, Theorem 2.4.3], every lift of $C$ ( component of $\left.\pi^{-1}(C)\right)$ in $\mathrm{H}$ is a prime, reduced cycle of length which is even times $|C|$. Note that $n$ is even.

Now, we choose a particular vertex $x$ such that $w(e)=-1$ for some arc $e$ with $o(e)=x$, and then consider a path from $x$ to any vertex $z$ in $G$. Let $C=\left(e_{1}, e_{2}, \cdots, e_{m}\right)$ be a $x$-cycle of $G$ such that $w\left(e_{1}\right)=-1$, where $e_{1}=(x, y)$. For any $j=1, \cdots, n$, let $\tilde{C}_{x_{j}}$ be a lift of $C$ in $H$ which is a $x_{j}$-cycle. Since each lift of $C$ has length even times $|C|$, we can let $\left|\tilde{C}_{x_{j}}\right|=2 k|C|$. Then we have

$$
\tilde{C}_{x_{j}}=\left(x_{j}, y_{\alpha\left(e_{1}\right)(j)}, \cdots, x_{\alpha(C)(j)}, \cdots, x_{\alpha^{2}(C)(j)}, \cdots, x_{\alpha^{2 k-1}(C)(j)}, \cdots, x_{j}\right) .
$$

Now, let $x_{j} \in \tilde{V}_{1}$ and $P=\left(x_{j}, y_{\alpha\left(e_{1}\right)(j)}, \cdots, x_{\alpha(C)(j)}\right)$ the subpath of $\tilde{C}_{x_{j}}$ with length $|C|$. Since $w(C)=-1, C$ has odd negative arcs in $G$, and so $P$ has odd negative arcs in $H$. Thus, we have $x_{\alpha(C)(j)} \in \tilde{V}_{2}$. Since $\left|\tilde{C}_{x_{j}}\right|=2 k|C|$, we have

$$
\left|\tilde{V}_{1} \cap\left\{x_{j}, x_{\alpha(C)(j)}, \cdots, x_{\alpha^{2 k-1}(C)(j)}\right\}\right|=\left|\tilde{V}_{2} \cap\left\{x_{j}, x_{\alpha(C)(j)}, \cdots, x_{\alpha^{2 k-1}(C)(j)}\right\}\right| .
$$

Therefore, it follows that

$$
\left|\tilde{V}_{1} \cap \pi^{-1}(x)\right|=\left|\tilde{V}_{2} \cap \pi^{-1}(x)\right| .
$$

Next, let $z \neq x$ be any vertex of $G$. Since $G$ is connected, there is a shortest $(x, z)$-path $Q=(x, \cdots, z)$ in $G$. By [5, Theorem 2.4.1], there are exactly $n$ lifts $\tilde{Q}_{x_{1}}, \cdots, \tilde{Q}_{x_{n}}$ such that $\tilde{Q}_{x_{j}}$ is a $\left(x_{j}, z_{\alpha(Q)(j)}\right)$-path for each $j=1, \cdots, n$.

The path $Q$ has either even or odd negative arcs in $G$. In either case, we have

$$
\left|\tilde{V}_{1} \cap \pi^{-1}(z)\right|=\left|\tilde{V}_{2} \cap \pi^{-1}(z)\right| .
$$

Therefore, it follows that

$$
\left|\tilde{V}_{1} \cap \pi^{-1}(v)\right|=\left|\tilde{V}_{2} \cap \pi^{-1}(v)\right|
$$


for any $v \in V(G)$.

Now, we construct a covering projection $\pi_{2}: H \longrightarrow G_{2}$. We project all vertices of $\tilde{V}_{1} \cap \pi^{-1}(v)$ and $\tilde{V}_{2} \cap \pi^{-1}(v)$ to a vertex $v_{1}$ and $v_{-1}$ of $G_{2}$, respectively.

Let $e=(x, y) \in D(G)$ such that $w(e)=-1$. By the above fact (1), there are $n / 2$ arcs $\tilde{e}_{1}, \cdots, \tilde{e}_{n / 2}$ from $\tilde{V}_{1}$ to $\tilde{V}_{2}$ and $n / 2$ arcs $\tilde{e}_{n / 2+1}, \cdots, \tilde{e}_{n}$ from from $\tilde{V}_{2}$ to $\tilde{V}_{1}$ as lifts of $e$ in $H$, where $H$ is an $n$-fold covering of $G$. Then, let

$$
\pi_{2}\left(\tilde{e}_{j}\right)= \begin{cases}e_{x_{1}}=\left(x_{1}, y_{-1}\right) & \text { if } j=1, \cdots, n / 2, \\ e_{x_{-1}}=\left(x_{-1}, y_{1}\right) & \text { if } j=n / 2+1, \cdots, n .\end{cases}
$$

Next, we consider an $e=(x, y) \in D(G)$ such that $w(e)=1$. Then there are $n / 2$ arcs $\tilde{e}_{1}, \cdots, \tilde{e}_{n / 2}$ from $\tilde{V}_{1}$ to $\tilde{V}_{1}$ and $n / 2$ arcs $\tilde{e}_{n / 2+1}, \cdots, \tilde{e}_{n}$ from from $\tilde{V}_{2}$ to $\tilde{V}_{2}$ as lifts of $e$ in $H$. Then, let

$$
\pi_{2}\left(\tilde{e}_{j}\right)= \begin{cases}e_{x_{1}}=\left(x_{1}, y_{1}\right) & \text { if } j=1, \cdots, n / 2, \\ e_{x_{-1}}=\left(x_{-1}, y_{-1}\right) & \text { if } j=n / 2+1, \cdots, n .\end{cases}
$$

Therefore, it follows that $\pi_{2}$ is a covering projection from $H$ to $G_{2}$.

Let $K$ be any intermediate covering to $H / G$ such that $\left(K, \tilde{w}_{K}\right)$ is balanced. Then $K$ is a covering of $G_{2}$, and so is an intermediate covering to $H / G_{2}$.

Conversely, let $K$ be an intermediate covering to $H / G_{2}$. Then, by $1,\left(K, \tilde{w}_{K}\right)$ is balanced, and $K$ is intermediate to $H / G$. Q.E.D.

If $(G, w)$ is balanced and $w^{-1}(1)=\phi$ (the empty set), then $G$ is a bipartite graph with partite sets $V_{1}, V_{2}$, where $V_{1} \cup V_{2}$ is a partition of $V(G)$ satisfying the condition of Theorem 6. By Theorem 7, we obtain the simple graph version of Theorem 4 in Stark and Terras $[16]$.

Corollary 4 Let $G$ be a simple connected graph with $\delta(G) \geq 2$. Suppose that $H$ is a connected bipartite covering of $G$. Then we have the following facts:

1. When $G$ is bipartite, every intermediate covering $\tilde{G}$ to $H / G$ is bipartite.

2. When $G$ is not bipartite, there is a unique quadratic covering $G_{2}$ intermediate to $H / G$ such that any intermediate covering $\tilde{G}$ to $H / G$ is bipartite if and only if $\tilde{G}$ is intermediate to $\mathrm{H} / \mathrm{G}_{2}$.

\section{Acknowledgment}

This work is supported by Grant-in-Aid for Science Research (C) in Japan. We would like to thank the referee for a lot of valuable comments and helpful suggestions. 


\section{References}

[1] H. Bass, The Ihara-Selberg zeta function of a tree lattice, Internat. J. Math. 3 (1992), 717-797.

[2] R. Feng, J. H. Kwak and K. I. Kim, Zeta functions of graph bundles, preprint.

[3] D. Foata and D. Zeilberger, A combinatorial proof of Bass's evaluations of the IharaSelberg zeta function for graphs, Trans. Amer. Math. Soc. 351 (1999), 2257-2274.

[4] J. L. Gross and T. W. Tucker, Generating all graph coverings by permutation voltage assignments, Discrete Math. 18 (1977), 273-283.

[5] J. L. Gross and T. W. Tucker, Topological Graph Theory, Wiley-Interscience, New York, (1987).

[6] F. Harary, On the notion of balance of a signed graph, Michigan Math. J. 2 (1955), 143-146.

[7] K. Hashimoto, Zeta Functions of Finite Graphs and Representations of p-Adic Groups, Adv. Stud. Pure Math. Vol. 15, pp. 211-280, Academic Press, New York, 1989.

[8] Y. Ihara, On discrete subgroups of the two by two projective linear group over p-adic fields, J. Math. Soc. Japan 18 (1966), 219-235.

[9] M. Kotani and T. Sunada, Zeta functions of finite graphs, J. Math. Sci. U. Tokyo 7 (2000), 7-25.

[10] H. Mizuno and I. Sato, Zeta functions of graph coverings, J. Combin. Theory Ser. B 80 (2000), 247-257.

[11] H. Mizuno and I. Sato, Weighted zeta functions of graphs, J. Combin. Theory Ser. B 91 (2004), 169-183.

[12] I. Sato, Decomposition formulas of zeta functions of graphs and digraphs, JP Jour. Algebra, Number Theory and Appl. 1 (3) (2001), 217-223.

[13] J. -P. Serre, Linear Representations of Finite Group, Springer-Verlag, New York, 1977.

[14] H. M. Stark and A. A. Terras, Zeta functions of finite graphs and coverings, Adv. Math. 121 (1996), 124-165.

[15] H. M. Stark and A. A. Terras, Zeta functions of finite graphs and coverings, part II, Adv. Math. 154 (2000), 132-195.

[16] H. M. Stark and A. A. Terras, Zeta functions of finite graphs and coverings, part III, to appear in Adv. Math.

[17] T. Sunada, L-Functions in Geometry and Some Applications, in "Lecture Notes in Math.", Vol. 1201, pp. 266-284, Springer-Verlag, New York, 1986.

[18] T. Sunada, Fundamental Groups and Laplacians(in Japanese), Kinokuniya, Tokyo, 1988. 